better decision-making and less restrictive care plans. The time it takes to write reports and attend tribunals seems a fair price to pay to ensure that those detained against their will have an effective right to challenge their situation.

1 Choong LS. The rise in the number of Section 2 detentions (letter). Psychiatrist 2011; 35: 198.

2 The NHS Information Centre. In-Patients Formally Detained in Hospitals under the Mental Health Act, 1983 and Patients Subject to Supervised Community Treatment: 1998-99 to 2008-09 (Appendix 2, Table 8). Health and Social Care Information Centre, 2009.

3 The NHS Information Centre, Community and Mental Health Team. InPatients Formally Detained in Hospitals under the Mental Health Act 1983 and Patients Subject to Supervised Community Treatment, Annual Figures, England 2009/10. Health and Social Care Information Centre, 2010 (http://www.ic.nhs.uk/pubs/inpatientdetmha0910).

4 Administrative Justice and Tribunals Council. Annual Report 2007/2008. TSO (The Stationery Office), 2008 (http://www.justice.gov.uk/ajtc/ docs/Annual_Report_2007_8.pdf).

Hugh Series is consultant psychiatrist and medical member of a first-tier mental health tribunal, Oxford Health NHS Foundation Trust and University of Oxford, UK, email: hugh.series@oxfordhealth.nhs.uk

doi: $10.1192 / \mathrm{pb} .35 .8 .315 b$

\section{Reader feedback is helpful, but are the leaflets readable?}

I was heartened to see an article evaluating the Royal College of Psychiatrists' patient information leaflets using quantitative and qualitative methods. ${ }^{1}$ The provision of information is critical to my clinical practice and has often involved these very leaflets. I was also pleased that the authors acknowledged that 'much patient information is written in complex language and is poorly presented' as these are often barriers to patients accessing information. Disappointingly, however, they did not conduct any analysis of the language; one respondent had commented regarding one leaflet that 'It has quite a high reading age'.

The complexity of language can be assessed using a range of readability measures such as Flesch Reading Ease (FRE; a document should have a score of greater than 60 , the higher the score the easier it is to read) and Flesch-Kincaid Grade Level (FKGL; refers to US school grades, so lower scores indicate better readability - a 13 year old should understand a document scoring 7). These are widely available, contained within word processing packages, and have been used to evaluate patient information leaflets in other specialties ${ }^{1}$ and standard appointment letters in child and adolescent mental health services. ${ }^{2}$ When these measures are applied to the College leaflets (Table 3 in the paper), the mean FRE is 7.81 (7.1-8.4) and mean FKGL 63.13 (58.7-69.8). This suggests the leaflets are readable as far as these computerised measures are concerned but their readability could be improved. When the top- and bottom-ranked leaflets (Table 3, which, curiously, has four highest ranked and three lowest ranked rather than four of each as described in the text) are compared, there is no statistical difference on either of the measures. This confirms that, although the language may be readable, the reader may not like the content.

I was confused by the quantitative method employed in the study. The original feedback was on a 5-point Likert scale ranging from 'strongly agree' to 'strongly disagree'. These are ordinal variables (variables which represent categories of a feature with some inherent ordering ${ }^{3}$ ); however, they were converted into continuous variables (one which can take any value within a range ${ }^{3}$ ) and analysed as such. Unfortunately, one cannot convert discrete categories into a linear scale in this way. Given this conversion, the values could only range $1-5$, and it is unsurprising that the authors found there was little variability in the feedback 'scores' assigned to each leaflet. It was also confusing to find that a correlation between modalities was included in the discussion but not presented in the results. My understanding of the analysis would have been aided to see the information presented in the original categories which those reading the leaflets had decided.

Despite these potential improvements and confusions, the conclusion remains undoubtedly true that 'reader feedback provides invaluable guidance about the substance and presentation of our public mental health information.' One can only hope that we continue to strive to produce information which is accessible to those who need it.

1 Briscoe M, Briscoe S, Timms P, Ramsay R. Usefulness of reader feedback on the Royal College of Psychiatrists' public information leaflets. Psychiatrist 2011; 35: 175-8.

2 Payne S, Large S, Jarrett N, Turner P. Written information given to patients and families by palliative care units: a national survey. Lancet 2000; 355: 1792.

3 Bennett DM, Gilchrist A. Readability of standard appointment letters. J Ment Health Fam Med 2010; 7: 101-6.

4 Harris M, Taylor G. Medical Statistics Made Easy (2nd edn). Scion, 2009.

Daniel M. Bennett, clinical lecturer in psychiatry and honorary specialty registrar in forensic psychiatry, University of Aberdeen, UK, email: danielm.bennett@nhs.net

doi: $10.1192 / p b .35 .8 .316$

\section{Readability analysis?}

As a trainee member of the Royal College of Psychiatrists' Public Education Editorial Board, I read with interest the review of reader feedback on the College online public education leaflets. ${ }^{1}$ I was struck by both the popularity of the public information section of the website and the high volume of completed feedback forms. I wondered, however, whether the authors have considered further analysis of the College information leaflets, to identify potential causes for the poorly scoring leaflets that they describe in the article.

The authors refer to an analysis of free-text feedback in which they name the two highest and lowest scoring main leaflets. It is perhaps unsurprising that poorly scoring leaflets would be more likely to receive negative comments, but what interested me most was the example constructive comment in response to the cannabis and mental health leaflet that said 'It has quite a high reading age'.

If the College information leaflets aim to reach a wide audience, it would seem sensible to establish whether the comment about reading age is in fact true for all leaflets. Is their readability consistent with the recommended level? And have the authors considered analysing whether there is a correlation between the reading age of the highest and lowest scoring leaflets? 